Research Paper

\title{
Biomimetic Acoustically-Responsive Vesicles for Theranostic Applications
}

\author{
Chen-Chan Hsieh, Shih-Tsung Kang, Yee-Hsien Lin, Yi-Ju Ho, Chung-Hsin Wang, Chih-Kuang Yeh and \\ Chien-Wen Chang ${ }^{\bowtie}$ \\ Department of Biomedical Engineering and Environmental Sciences, National Tsing Hua University, Hsinchu, 30013, Taiwan \\ $\bowtie$ Corresponding author: E-mail: chienwen@mx.nthu.edu.tw \\ (C) 2015 Ivyspring International Publisher. Reproduction is permitted for personal, noncommercial use, provided that the article is in whole, unmodified, and properly cited. \\ See http://ivyspring.com/terms for terms and conditions.
}

Received: 2015.02.10; Accepted: 2015.07.05; Published: 2015.09.08

\begin{abstract}
In recent years, biomimetic cell membrane-derived particles have emerged as a new class of drug delivery system with advantages of biocompatibility, ease of isolation and long circulation profile. Here we report the development and potential theranostic applications of a new biomimetic acoustically-responsive droplet system derived from mammalian red blood cell membrane (RBCM). We hypothesized that drug-loaded RBCM droplets (RBCMDs) would undergo a transition from liquid (droplets) to gas (bubbles) upon high intensity focused ultrasound (HIFU) insonation, resulting in on-demand drug release. The generated microbubbles could also serve as a contrast agent to enhance ultrasound imaging. As-synthesized RBCMDs exhibited uniform size, good dispersity and preservation of RBCM-associated proteins that prevented uptake by macrophages. Camptothecin (CPT), an anti-cancer drug, was successfully loaded in the RBCMDs with a loading efficiency of $2-3 \%$ and an encapsulation efficiency of $62-97 \%$. A short ( $3 \mathrm{~min}$ ) exposure to HIFU irradiation triggered release of CPT from the RBCMDs and the physical explosion of droplets damaged nearby cancer cells resulting in significant cell death. In addition, the acoustically vaporized RBCMDs significantly increased the ultrasound echo signal to $30 \mathrm{~dB}$. Lastly, we demonstrated that RBCMDs could be acoustically vaporized in vivo in target tissues, and enhancing ultrasound imaging. Taken together, we have developed a new class of naturally derived RBCMDs which show great potential for future application in remotely triggered drug delivery and ultrasound imaging enhancement.
\end{abstract}

Key words: red blood cell membrane, ultrasound theranostics, remotely triggerable drug delivery, cancer therapy, biomimetic vesicles.

\section{Introduction}

Synthetic materials camouflaged inside naturally-derived membranes show great promise for cancer-targeting drug delivery $[1,2]$, in vivo detoxification treatment [3] and prolonged in vivo circulation [4]. As the most robust source for preparing biomembranes, red blood cells (RBCs) are abundant long-circulating oxygen carriers and can be quantitatively isolated from the body. Their intrinsic biocompatibility and non-immunogenicity allow them to stably circulate in the blood for 120 days. These remarkable properties have inspired the design of long-circulating RBC-mimicking drug delivery systems aimed at in- creasing drug availability to targeted organs or tissues [5]. Various RBC-based drug delivery systems including cargo-loaded carrier RBCs [6, 7], synthetic delivery vehicles mimicking natural RBCs $[8,9]$, RBC membrane-derived liposomes [10] and RBC membrane-camouflaged nanoparticles $[4,11,12]$ are currently undergoing preclinical or clinical development.

Recently, the concept of external stimuli-responsive drug delivery has gained increased attention due to its capability of spatially- and temporally-accurate drug release. Triggerable systems could be designed to provide safer and more effective drug 
delivery compared to conventional methods. To attain a triggerable delivery system, various external stimuli such as near infrared (NIR)-mediated photothermal effects [13, 14], ultrasound [15] and magnetic hyperthermia [16], have been successfully attempted. Among the various commonly used external stimuli, ultrasound possess advantageous on its non-invasive nature and high tissue-penetrability [17-20]. Conventional acoustic vehicles made of low boiling points compounds (i.e. perfluorocarbon) stabilized by synthetic lipids have been explored for their potential on drug delivery [21], gene delivery [22], anti-cancer therapy [23] and ultrasound imaging [19]. However, utilization of autologous-derived RBC for the fabrication of ultrasound-responsive acoustic vehicles has not been attempted yet, despite the potential of this novel biomimetic platform to overcome drawbacks associated with conventional drug delivery designs. The goal of this study was to develop a biomimetic RBC membrane-derived droplet (RBCMD) system by

(A)

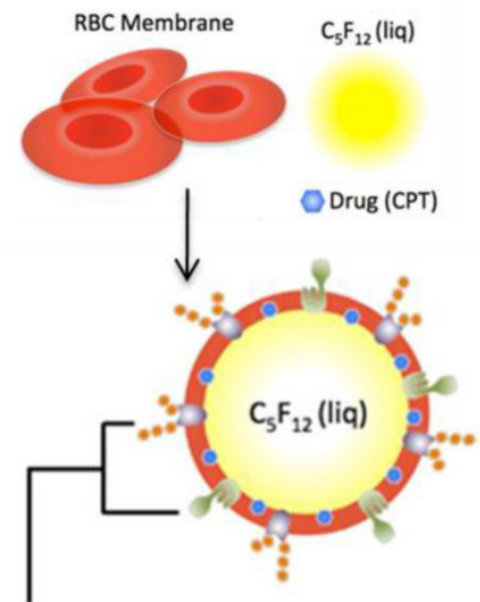

(B)
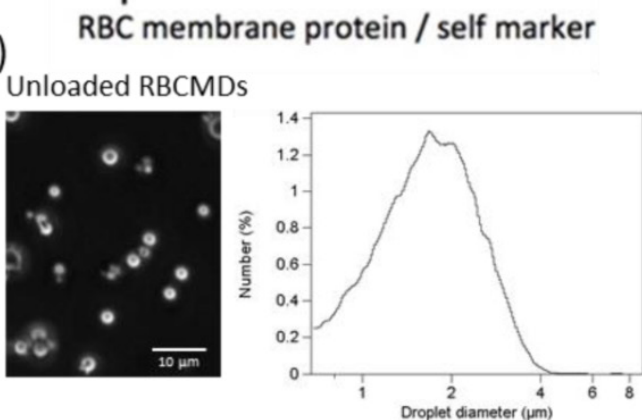

$200 \mu \mathrm{g} / \mathrm{mL}$ CPT-fed RBCMDs
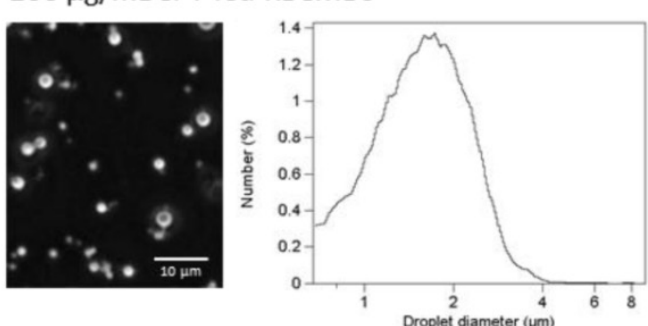

combining the advantages of biocompatible RBC-derived material with the strength of ultrasound-triggered drug delivery.

We designed biomimetic RBC droplets containing a perfluoro-n-pentane $\left(\mathrm{C}_{5} \mathrm{~F}_{12}\right.$, boiling point: $\left.29^{\circ} \mathrm{C}\right)$ liquid core with a drug-loaded RBC membrane shell (Figure 1A). The synthesized RBCMDs exhibited good biocompatibility and decreased uptake by macrophages. We hypothesized that high intensity focused ultrasound (HIFU) insonation would induce violent vaporization of $\mathrm{C}_{5} \mathrm{~F}_{12}$ in RBCMDs which would impair the integrity of the RBC membrane and release the drug. The physical force generated during the process of droplet vaporization may further promote drug distribution into the interstitial region of a tumor by disrupting local vascular endothelial junctions. In addition, the generated bubbles could serve as an imaging contrast agent because of their high acoustic impedance.

(C)

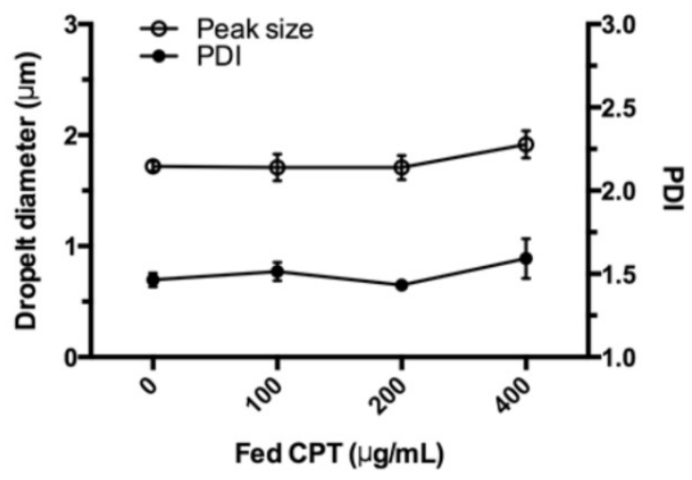

$100 \mu \mathrm{g} / \mathrm{mL}$ CPT-fed RBCMDs

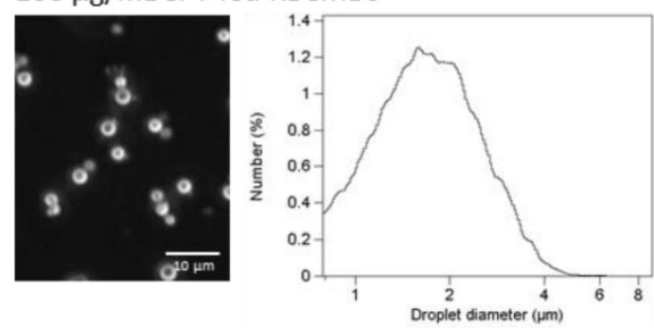

$400 \mu \mathrm{g} / \mathrm{mL}$ CPT-fed RBCMDs
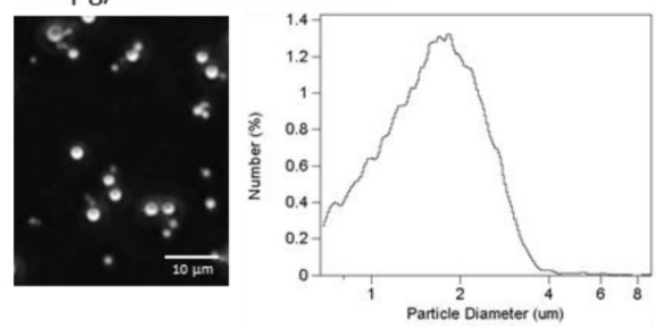

Figure 1. (A) Schematic representation of RBCMDs preparation. (B) Microscopic images and size distribution of CPT-loaded RBCMDs. (C) Particle size and polydispersity index (PDI) of CPT-loaded RBCMDs. Size distribution was measured with a Multisizer 3 Coulter counter. Data represent the mean \pm S.D.; $n=3$. 


\section{Results and Discussion}

Mouse RBCs were treated with hypotonic media to obtain RBC membrane (RBCM) ghosts. These RBC ghosts were used to fabricate drug-loaded RBCMDs by mild sonication in an ice bath. The sonication power was adjusted to efficiently break up the organic phase (perfluoro-n-pentane) into microdroplets suspended in a continuous aqueous phase containing RBC-derived ghosts. The resultant microdroplets were immediately covered and stabilized by $\mathrm{RBC}$ membrane shells. Camptothecin (CPT), a hydrophobic model drug, was successfully encapsulated within the lipid shell in the RBCMDs. The as-prepared RBCMDs (with or without CPT) exhibited good dispersity and uniform size (Figure 1B, C). The average size of RBCMDs was approximately $1.7 \mu \mathrm{m}$ and $3 \sim 5 \times$ $10^{9}$ droplets $/ \mathrm{mL}$ could be prepared using $1 \mathrm{~mL}$ of mouse blood. Retention of RBCM-associated proteins by RBCMDs was assessed by SDS-PAGE analysis. The overall protein profile was similar in extracts from RBCMDs and fresh RBCMs (Figure 2A). This prelim-

(A)

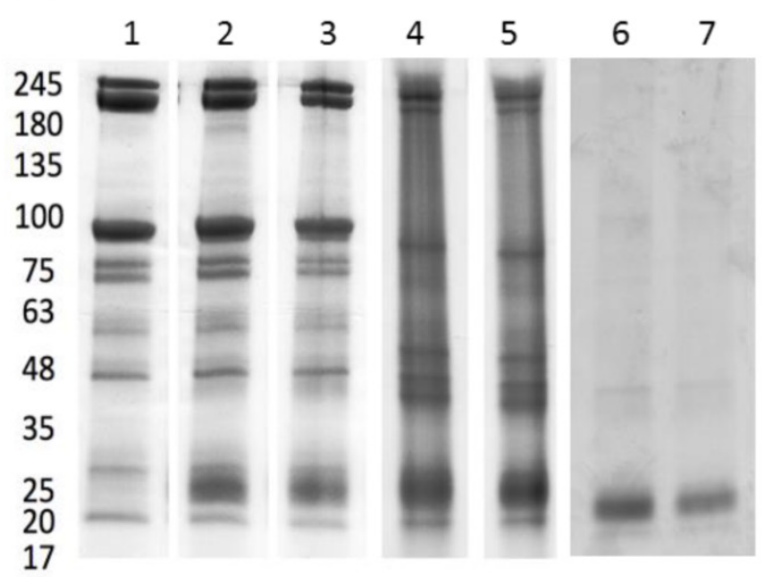

(B)
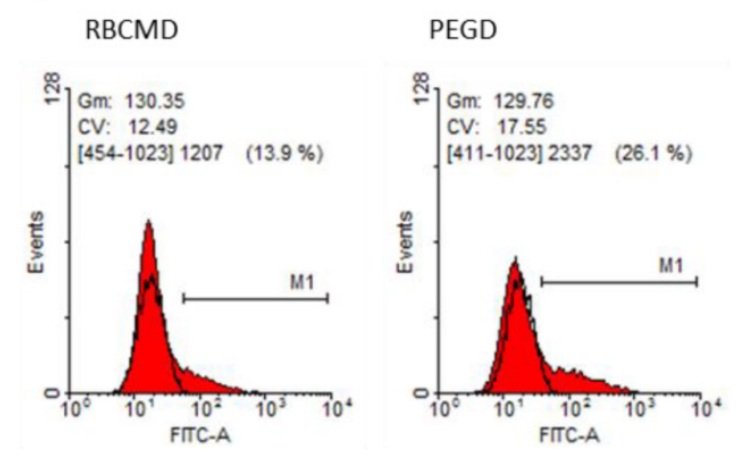

inary evaluation suggested that the optimized sonication procedure did not cause dramatic loss of proteins from the RBC membrane.

One of the main obstacles to systemic drug delivery is the rapid clearance of injected drugs from circulation, which often results in poor bioavailability and sub-optimal therapeutic outcomes. It has been suggested that the majority of injected drugs are prone to opsonization in the blood and subsequent phagocytotic uptake by macrophages in the reticuloendothelial system (RES) of the liver and spleen. Kupffer cells, a specialized type of macrophages found in the liver RES, are capable of actively ingesting circulating "foreign" substances on the basis of molecular recognition. In contrast, self-derived RBCs appear to avoid the phagocytotic activities of Kupffer cells and this self-recognition mechanism has inspired their development as long-circulating vehicles. In this study, isolated mouse primary macrophages were used as an in vitro model to test whether our sonication-fabricated RBCMDs maintained the ability to avoid macrophage uptake, as suggested by their sim-

(C)

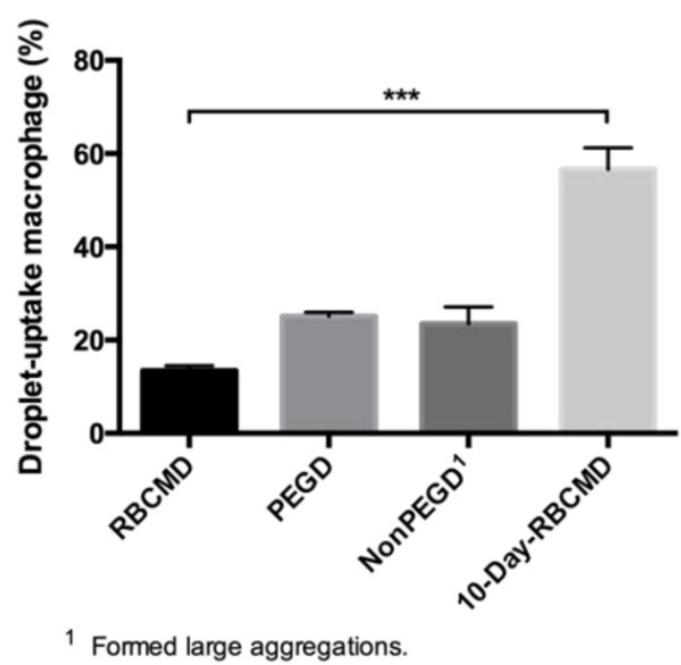

NonPEGD 10-day-RBCMD
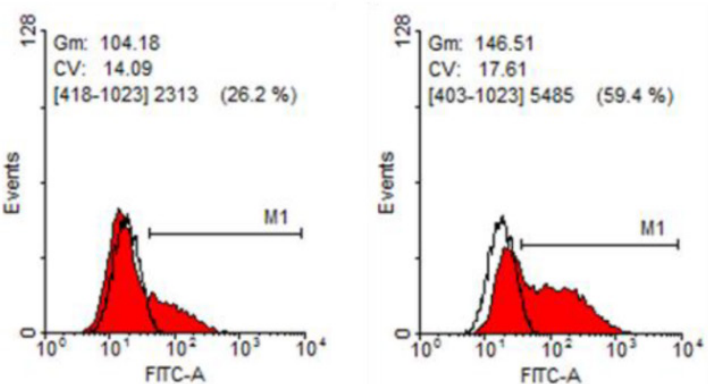

Figure 2. Macrophage uptake of RBCMDs and DSPC-based droplets after $10 \mathrm{~min}$ of incubation. (A) Membrane protein preservation of RBCMDs under various storage conditions. Sample 1-5: w/ protease inhibitors. Sample 6-7: w/o protease inhibitors. 1: fresh RBCM; 2: fresh RBCMDs; 3: 1-day RBCMDs; 4: 4-day RBCMDs; 5: 10 day-RBCMDs; 6: 10-day RBCM; 7: 10-day RBCMDs. (B) Flow cytometric analysis of droplet uptake by mouse primary macrophages. RBCMD: fresh RBCMDs; PEGD: PEG-modified DSPC droplets; NonPEGD: Unmodified DSPC droplets; 10-day-RBCMD: 10 days old RBCMDs. (C) Quantitative results of uptake of various droplets by mouse macrophages. Data represent the mean \pm S.D.; $n=3$. 
ilar protein composition to RBCMs. Mouse primary peritoneal macrophages were freshly isolated and incubated with different kinds of droplets, including: (1) freshly-prepared RBCMDs, (2) 10-day-old RBCMDs, (3) polyethylene glycol (PEG)-modified 1,2-distearoyl-OT-glycero-3-phosphocholine (DSPC) droplets, and (4) unmodified DSPC droplets as a control. The results showed dramatically different macrophage uptake rates analyzed using fluorescence-activated cell sorting (FACS) (Figure 2B). After 10 minutes of co-culturing, PEG-modified DSPC droplets and fresh RBCMDs had lower uptake rates ( $25.1 \%$ and $13.5 \%$, respectively) compared to the high uptake $(56.6 \%)$ of 10-day-old RBCMDs (Figure 2C). Unmodified DSPC droplets were taken up at a rate of $23.5 \%$, but they formed large aggregates $(>50 \mu \mathrm{m})$ in the culture medium, likely interfering with their uptake by macrophages. The results demonstrated that RBCMDs and PEG-modified droplets could effectively maintain good dispersity and avoid macrophage uptake. In addition, freshly prepared RBCMDs were more resistant to macrophage uptake than 10-day-old RBCMDs. This observation emphasized the importance of using fresh RBCMDs to achieve long circulation. We suspected that the distinct macrophage uptake between fresh and 10-day-old RBCMDs might be due to the differential protein preservation or stability on RBCMDs after different storage times. Preservation of total membrane protein on RBCMDs under different storage conditions was examined using SDS-PAGE analysis. The results (Figure 2A) show that the membrane proteins were best preserved in the presence of protease inhibitors at $4^{\circ} \mathrm{C}$ within 1 day. Under the same storage condition, dramatic changes on the total protein profile were observed after 4 days. Maximal total protein degradation was observed from RBCMDs stored at $4^{\circ} \mathrm{C}$ for 10 days without protease inhibitors. Based on the results, we conclude that protein preservation on RBCMDs was dependent on the storage time as well as the use of protease inhibitors. It has been previously suggested that suppression of the phagocytotic activity of macrophages may occur through the interaction of CD47, located on the surface of the RBCM, with macrophage SIRP $\alpha$ (Signal-regulatory protein alpha) [24]. In this study, the preservation of CD47 (marker of self) on RBCMDs was further analyzed using Western Blot method. The results (Additional File 1: Figure S1) clearly show that CD47 was detected from both fresh RBCM and RBCMDs but not from the 10-day-old RBCM and RBCMDs. Our results confirmed the significance of CD47 presentation on RBCMDs to avoid macrophage uptake. The presentation of CD47 on fresh RBCMDs may account for its lower macrophage uptake comparing to the 10-days-old RBCMDs.

We investigated the potential of applying RBCMDs as a triggerable drug delivery carrier for cancer therapy. CPT is a hydrophobic compound which exhibits strong anti-neoplastic effects on colon, lung, breast, stomach and ovarian cancers [25]. To fabricate CPT-RBCMDs, CPT was dissolved in dimethyl sulfoxide (DMSO), and added into the $\mathrm{RBCM} / \mathrm{PFC}$ mixture prior to sonication. At initial CPT concentrations of 100, 200 and $400 \mu \mathrm{g} / \mathrm{mL}$, the CPT loading efficiency (L.E.) into RBCMDs was $0.87 \pm$ $0.16 \%, 1.95 \pm 0.29 \%$ and $3.37 \pm 0.72 \%$, and the CPT encapsulation efficiency (E.E.) was $102.55 \pm 12.57 \%$, $95.28 \pm 6.93 \%$ and $80.87 \pm 6.19 \%$, respectively (Table 1). As initial CPT concentration was increased, L.E. gradually increased whereas E.E. decreased. At higher concentrations of initial CPT $(>200 \mu \mathrm{g} / \mathrm{mL})$, the resultant RBCMDs were less stable and prone to vaporization before HIFU insonation. This phenomenon may be explained by destabilization of RBCM shells after incorporation of excess CPT. Since RBCMDs fabricated with $100 \mu \mathrm{g} / \mathrm{mL}$ of CPT showed better stability, they were used for further studies. As a potential triggerable drug delivery system, sufficient colloidal stability with minimal drug leakage is desired for RBCMDs. The stability of RBCMDs was tested at $37^{\circ} \mathrm{C}$ in serum to mimic the in vivo environment. Particle stability of RBCMD was comparable to the conventional acoustic droplets prepared by synthetic lipids for at least 4 hours then decreased with time (Figure 3). The decrease in droplet concentration was presumably due to the coalescence and Oswald ripening of relatively unstable droplets, as indicated by the slight increase in droplet size. The enlarged droplets were less stable due to decreased Laplace pressure, and so were more likely to undergo spontaneous vaporization. Generally, when applying lipid-based microbubbles or microdroplets for US-triggered drug delivery applications, the intravenous-injected microbubbles or microdroplets usually receive US within 1 hour after their administration [26]. Thus, based on the results, the stability of RBCMDs might be sufficient for further US treatments in vivo.

Table 1. Loading efficiency (L.E.) and encapsulation efficiency (E.E.) of CPT-loaded mouse RBCMDs. Data represent the mean \pm S.E.; $\mathrm{n}=3$.

\begin{tabular}{lll}
\hline Initial CPT $(\mu \mathrm{g} / \mathrm{mL})$ & Loading efficiency $(\%)$ & Encapsulation efficiency $(\%)$ \\
\hline 100 & $0.87 \pm 0.16$ & $102.6 \pm 12.57$ \\
200 & $1.95 \pm 0.29$ & $95.28 \pm 6.93$ \\
400 & $3.37 \pm 0.72$ & $80.87 \pm 6.19$ \\
\hline
\end{tabular}


(A)

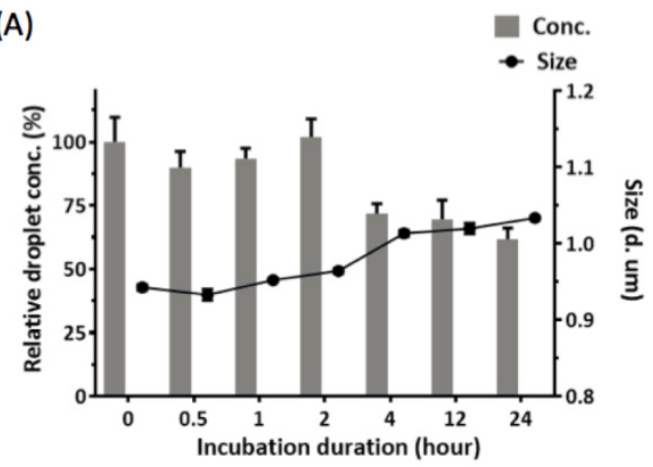

(B)

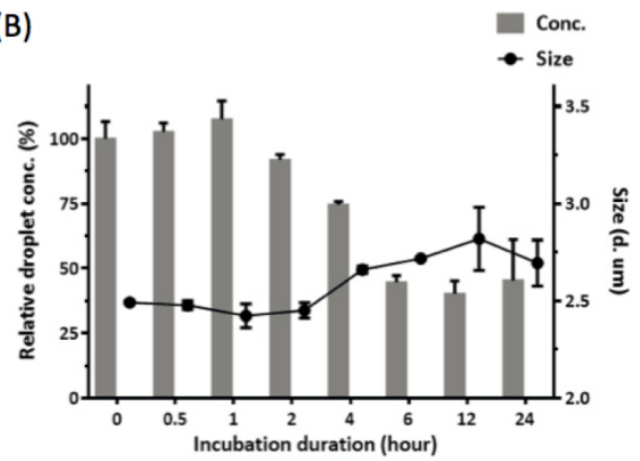

Figure 3. Colloidal stability of RBCMDs at $37 \circ \mathrm{C}$ in serum. After the predesignated incubation lengths, droplets were resuspended and examined for the concentration and size by using a Multisizer. The relative droplet concentration was determined by comparing the measured droplet concentration to the initial droplet concentration. (A) PEG-modified DSPC droplets. (B) RBCMDs. Data represent the mean \pm S.D.; $n=3$.
Next we investigated whether RBCMDs could undergo acoustic vaporization and facilitate ultrasound imaging. Acoustic vaporization of RBCMDs by HIFU insonation was verified by bubble generation (Figure 4A). B-mode ultrasound examination of acoustic RBCMD vaporization showed a low echo signal from RBCMDs in droplet form, but after acoustic droplet vaporization (ADV), the perfluoro-n-pentane inside the RBCMDs was vaporized to produce bubbles that significantly increased the detected B-mode echo signal (Figure 4B). Since droplet-to-bubble conversion was accompanied by a five-fold expansion in the diameter and enhancement of acoustic impedance relative to the surrounding medium, vaporized RBCMDs produced much higher contrast enhancement in ultrasound images than droplet-form RBCMDs [27, 28]. Quantitative analysis of the signal to noise ratio (SNR) of the ultrasound echo signal is shown in Figure 4C. The SNR was lower than $15 \mathrm{~dB}$ when RBCMDs were not vaporized. After acoustic vaporization, we detected a significant enhancement of in the SNR. The post-ADV SNR values continued to increase as the droplet concentration was increased from 0 to $64 \times 10^{6}$ droplets $/ \mathrm{mL}$, demonstrating the feasibility of using RBCMDs for ultrasound imaging. The high echogenicity, good SNR and controllable activation of RBCMDs illustrate their strong potential for clinical applications in early stage diagnosis and treatment of cancer.

\section{(A)}

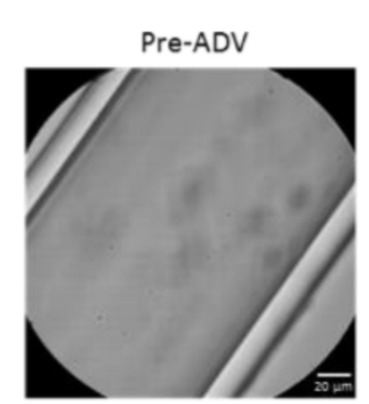

Post-ADV

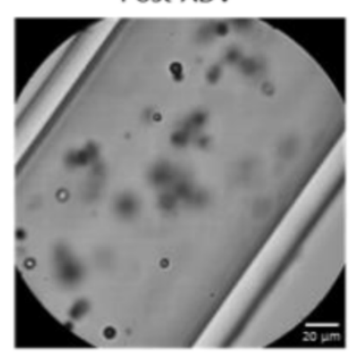

(B)
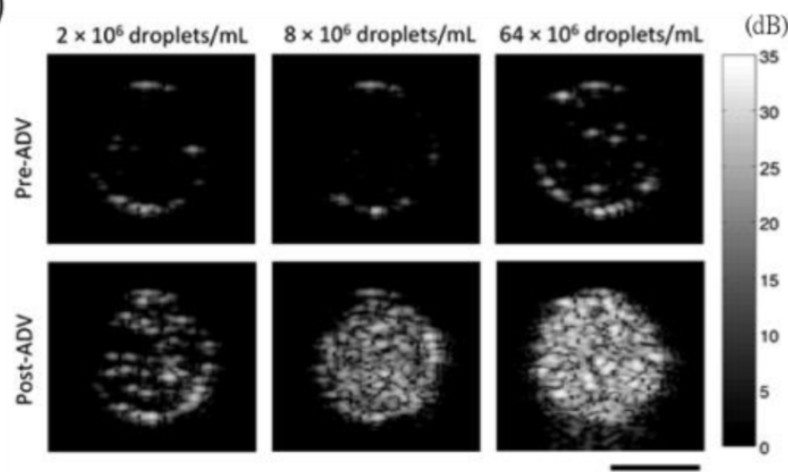

(C)

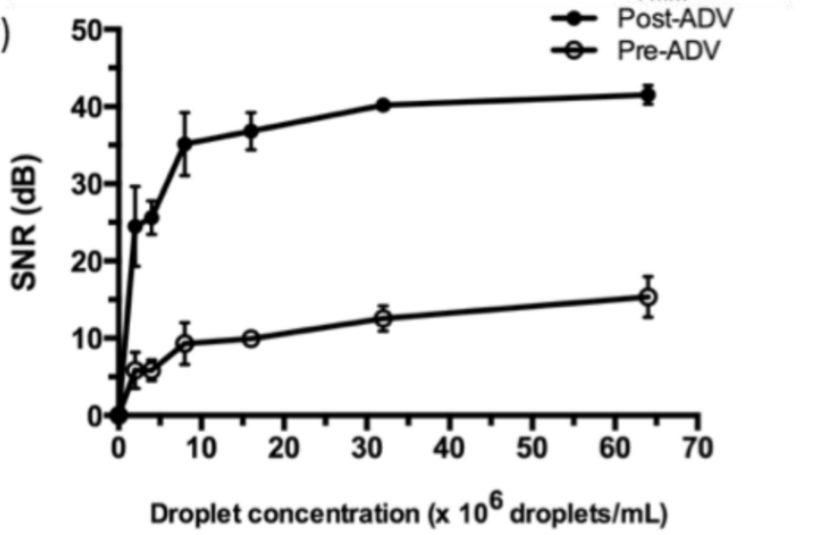

Figure 4. Acoustic vaporization of RBCMDs for ultrasound B-mode imaging. (A) Microscopic images of RBCMDs before and after ADV. (B) B-mode ultrasound imaging of RBCMDs before and after ADV. (C) Quantitative signal to noise ratio of B-mode imaging. Data represent the mean \pm S.D.; $n=3$. 
Besides their imaging function, the therapeutic potential of RBCMDs as novel anti-cancer drug delivery carriers was explored in vitro. Before receiving ultrasound treatment, accumulative drug leakage from RBCMDs was minimal ( $<10 \%)$ even after 4 hours incubation at $37^{\circ} \mathrm{C}$ (Additional File 1: Figure S2A). Upon receiving HIFU treatment, around 40\% CPT was released from CPT-RBCMDs (Additional File 1: Figure S2B). Next, the effects of various treatments, including: RBCMDs, US, CPT-RBCMDs, RBCMDs+US and CPT-RBCMDs+US on the viability of human cervical cancer cells (Hela) were studied (Figure 5). Cell viability was determined using MTT assay 24 hours after the designated treatments. High viability $(>95 \%)$ was maintained for the cells treated with RBCMDs, ultrasound or CPT-RBCMDs alone. The results indicate that either RBCMDs or US alone did not cause detrimental effects on cell viability. Interestingly, without HIFU irradiation, CPT-RBCMDs did not cause significant cell death. In contrast, high cancer cell death was observed from cells received RBCMDs+US (viability $50 \%$ ) or CPT-RBCMDs+US (viability $30 \%$ ) treatment respectively. It was likely that the vaporization of RBCMDs exerted cytotoxic physical impacts to the neighboring cells. Furthermore, when applying US with the drug-loaded RBCMDs (CPT-RBCMDs), the released CPT can exert chemotherapeutic effects. To elucidate the mechanism in details, the physical or chemical effects of combining US with CPT-RBCMDs on cancer cell death was examined separately next.

(A)

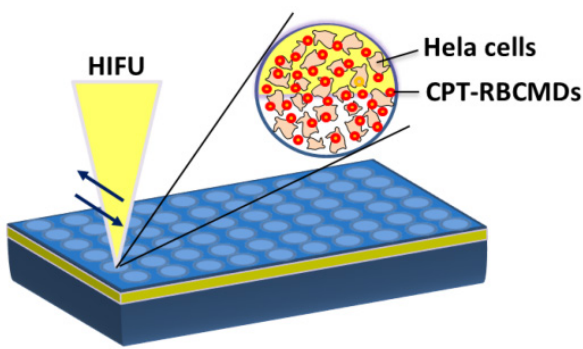

(B)

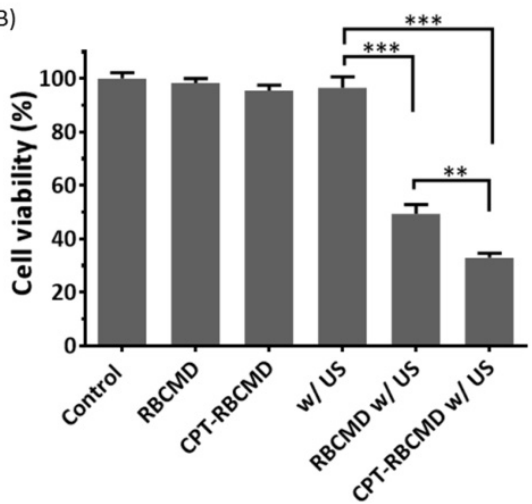

Figure 5. Anti-cancer efficacy by the combined treatment of ultrasound/CPT-RBCMDs. (A) Experimental scheme. (B) Hela cells were seeded in 96 well plates and received the following treatments: control, RBCMD, CPT-RBCMD, ultrasound, RBCMD + ultrasound, CPT-RBCMD + ultrasound. After the treatments, cell viability was analyzed after 24 hour incubation by MTT assay.
To investigate the effects of physical impact generated from RBCMDs received HIFU irradiation, an integrated acousto-optical system [29] was used to closely monitor damage of cancer cells caused by the vaporization of RBCMDs. RBCMDs were co-incubated with BJAB cancer cells and subjected to ultrasound irradiation. Obvious damage to cell integrity caused by RBCMD acoustic vaporization was observed (Figure 6A, Additional File 2: Video S1). As a control, treatment with RBCMDs alone did not cause any deleterious effects on cell viability and integrity. After ultrasound treatment, the cells were re-plated to grow. The resultant cell number was significantly less in the experimental group (RBCMD+US) compared to other control groups (Figure 6B). The effects of acoustic RBCMD vaporization on cell viability were subsequently quantitated by MTT assay (Figure 6C). A significant drop in cell viability (over $50 \%$ ) was observed in BJAB cells treated with RBCMDs and subjected to ultrasound insonation for 3 minutes, indicating efficient ADV of RBCMDs to damage nearby cancer cells. We note that the approximately $20 \%$ decline in viability in the ultrasound-only treated group (w/o RBCMD) was likely due to the cavitation effects of dissolved gas bubbles in the culture medium.

The chemical effects of ultrasound-triggered drug release of CPT-RBCMDs on cancer cell death were examined next. After 3 minutes of exposure to HIFU insonation, intensive droplet vaporization was accompanied by elevated release of CPT in the medium, as detected by spectroscopy (Figure 7B). Cumulative release of CPT was well correlated with the initial droplet concentration, indicating effective ultrasound-mediated drug release. The CPT release efficiency remained nearly at the same level of 30-40\% with different droplet concentrations from $1 \times 10^{8}$ to 4 $\times 10^{8}$ droplets $/ \mathrm{mL}$ (Figure $7 \mathrm{C}$ ). The anti-cancer effects of released CPT were examined by adding the released CPT into the cell culture medium and observing the viability changes of Hela cells after 24 hours of incubation. The cell viability of Hela cells dramatically decreased after they were incubated with CPT-containing supernatant from HIFU-treated CPT-loaded RBCMDs (Figure 7D). In contrast, the control group (treated with supernatant from the CPT loaded-RBCMDs without HIFU insonation) did not show a significant change in cell viability. Lastly, we examined whether RBCMDs could be acoustically vaporized to produce ultrasound contrast enhancement in vivo. Male C57BL mice were injected with a bolus of diluted RBCMDs through the retro-orbital sinus and were subsequently placed for $10 \mathrm{~min}$ to ensure even distribution of RBCMDs in the circulation. Afterward, pulsed HIFU was applied to the leg 
muscle for 2 min to induce ADV, during which ultrasound B-mode imaging was performed to monitor the occurrence of bubbles. The results showed that ultrasound contrast enhancement indicative of the presence of bubbles was readily detected in the leg muscle after HIFU exposure (Figure 8), suggesting

(A)
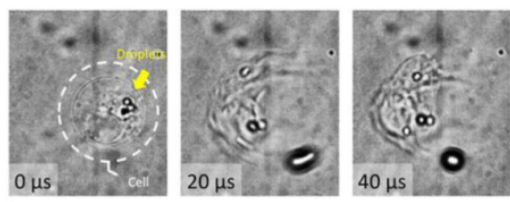

(C) that RBCMDs can undergo in vivo ADV. The on-demand droplet-to-bubble conversion suggests RBCMDs' potential for use as a theranostic agent for real-time imaging during an ultrasound therapeutic process that needs a relatively long time course.
(B)

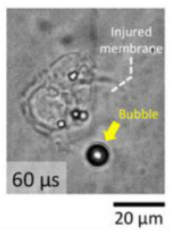

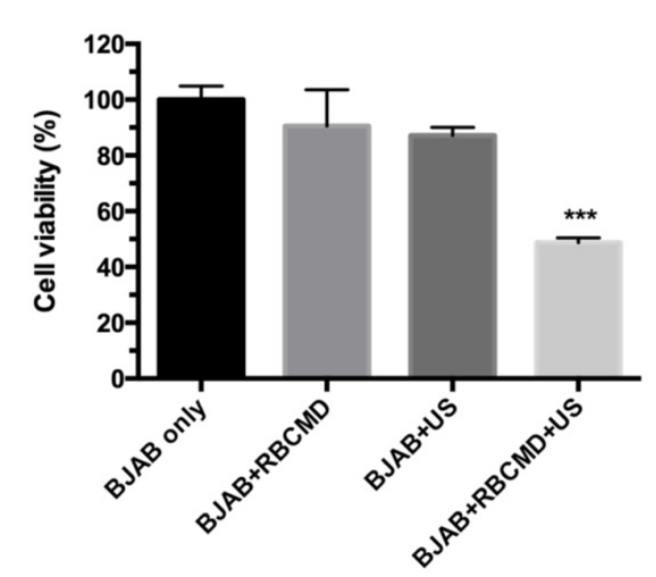

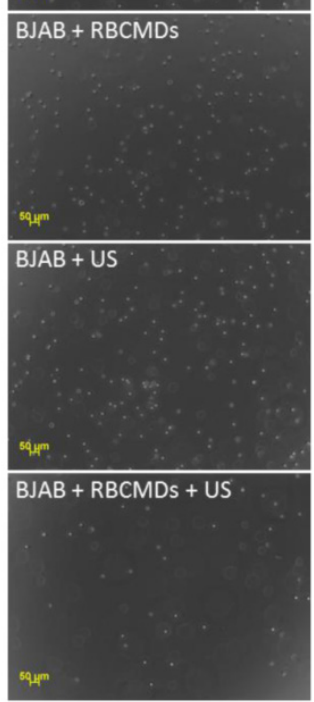

Figure 6. Cancer cell killing by physical damage from acoustic vaporization of RBCMDs. (A) Physical damage of a BJAB cell by acoustic vaporization of RBCMDs was observed using a high-speed camera. (B) Morphology of BJAB cells after the designated treatments, including 3 minutes of ultrasound (cells only, cells + RBCMDs, cells + US, cells + RBCMDs + US). (C) The viability of BJAB cells after the designated treatments (cells only, cell + RBCMDs, cells +US, cells + RBCMDs + US) was quantitated using an MTT assay. Data represent the mean \pm S.D.; $n=3$.

(A)

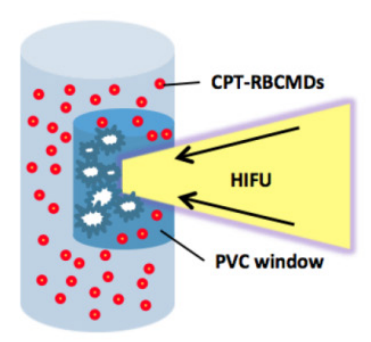

(C)

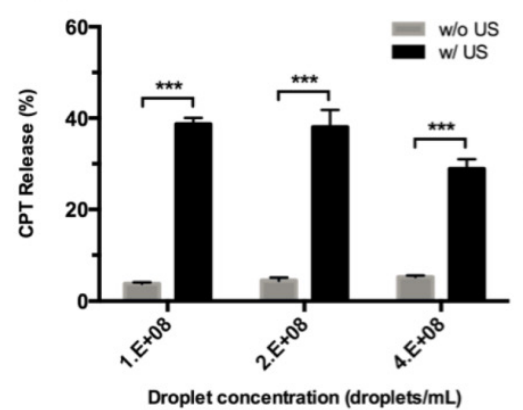

(B)

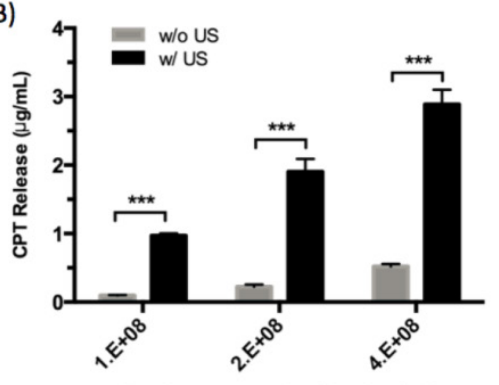

(D)

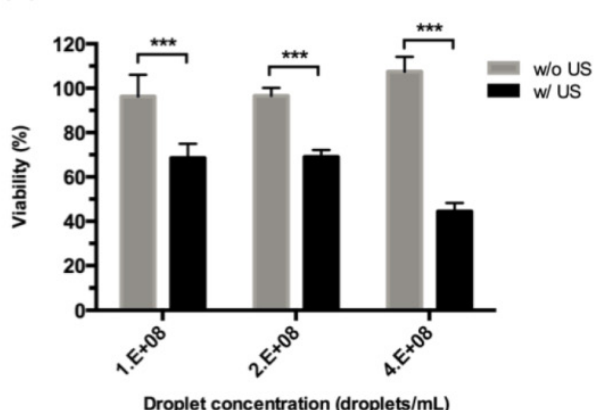

Figure 7. Drug (CPT) release from CPT-RBCMDs by ultrasound treatment and its anti-cancer cell effects. (A) Schematic representation of HIFU-triggered drug release from CPT-RBCMDs. (B) CPT released at various CPT-RBCMDs concentrations. (C) Percentage of CPT release at various CPT-RBCMDs concentrations. (D) Viability of Hela cells treated with acoustically released CPT and incubated for $24 \mathrm{hr}$. Cell viability was determined using an MTT assay. Data represent the mean \pm S.D.; $\mathrm{n}=3$. 

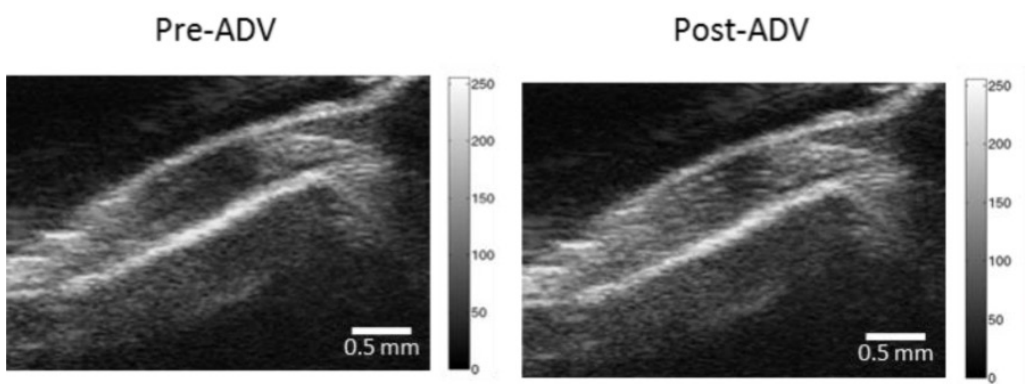

Color-coded contrast enhancement

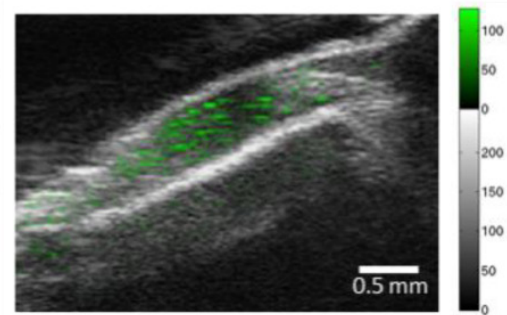

Figure 8. In vivo acoustic vaporization of RBCMDs. Pre-ADV and post-ADV ultrasonic B-mode images of a muscle region. The color-coded contrast enhancement (right image) represents the spatial distribution of ADV bubbles.

\section{Conclusion}

In this study, we demonstrated the successful preparation and evaluation of a biomimetic remotely-triggerable drug delivery system (RBCMDs) based on a naturally-derived RBC platform. The as-prepared RBCMDs displayed good in vitro biocompatibility and minor uptake by macrophages. An effective in vitro anti-cancer cell effect was observed by either ultrasound-triggered vaporization or US-triggered drug release from the RBCMDs. The capability of acoustic RBCMD vaporization could also provide useful ultrasound contrast imaging. To further promote the clinical translatability of RBCMDs, the following issues may be taken into consideration. First, all the bio-derived materials need to receive comprehensively sterilization procedure to prevent potential contamination. Second, the blood type matching should be performed in case the RBCM was collected from a non-autologous origin. Nonetheless, considering the ease of rapid fabrication, biomimetic properties and potential drug delivery/molecular imaging capabilities, we anticipate RBCMDs is worth of further development for its clinical theranostic applications.

\section{Materials and Methods}

\section{Preparation of RBC membrane}

RBC membrane was prepared following the previous published protocols with modifications [4]. The whole blood was first withdrawn from male C57BL mice (6-8 w) obtained from National Laboratory Animal Center (NLAC, Taiwan) through cardiac puncture using a syringe containing EDTA solution. After low speed centrifugation for $5 \mathrm{~min}$ at $4{ }^{\circ} \mathrm{C}$, the plasma and buffy coat were carefully removed. The resulting packed RBCs were washed three times with ice cold PBS following by re-suspending in $0.25 \times$ PBS (hypotonic medium) in an ice bath for $30 \mathrm{~min}$ for hemolysis. To remove components in cytoplasm, sedimented RBCs were resuspended with hypotonic solution in an ice bath and were pelleted by centrifuging at $10,000 \times g$ for $40 \mathrm{~min}$. The RBC membrane pellet was obtained and verified using microscopy. All the buffers used in the RBC membrane preparation procedure were sterile and contained protease inhibitors ( $1 \mathrm{ug} / \mathrm{mL}$ Aprotinin; $50 \mathrm{uM}$ Leupeptin).

\section{Fabrication of RBC membrane-derived drop- lets (RBCMDs)}

The mixture containing RBC membrane, glycerol and perfluoro-n-pentane was prepared in PBS on ice (For fabricating CPT or DiO loaded RBCMDs, CPT or $\mathrm{DiO}$ was dissolved in 10\% DMSO and added into the lipid/PFC mixture). After brief vortex, the mixture was sonicated for $15 \mathrm{sec}$ on ice using probe sonicator (VCX 750, Sonics, CT, USA). Formed droplets were subjected to three washing/centrifugation cycles and resuspended with fresh PBS to remove the free components. The as-prepared RBCMDs were stored at $4{ }^{\circ} \mathrm{C}$ before further uses. All the buffers used in the above procedure were sterile and contained protease inhibitors (1 ug/mL Aprotinin; $50 \mathrm{uM}$ Leupeptin). Morphology of RBCMDs was observed by optical microscopy. Droplet concentration and size distribution were measured using electrical sensing zone system (ESZ) with a Multisizer 3 Coulter counter (Beckman Coulter, CA, USA). Polydispersity index (PDI) was calculated as the volume-weighted mean diameter divided by the number-weighted mean diameter. Previous study by Feshitan and coworkers has showed that PDI for the 1-2 $\mu \mathrm{m}$ isolated-microbubbles was about 1.5 and which similar to our results.[30]

\section{Membrane protein retention}

Samples of RBC membrane and RBCMDs were prepared in SDS sample buffer (Invitrogen). Proteins of the samples were analyzed by $4-15 \%$ Mini-PROTEAN precast gels (BIO-RAD) in Tris-Glycine running buffer with 120 Voltage for 70 min. The resulting polyacrylamide gel was stained in coomassie brilliant blue for protein visualization. 


\section{CPT loading efficiency and encapsulation effi- ciency}

Encapsulated CPT concentration was determined from the fluorescent intensity of CPT. Methanol was first added to the droplet solutions and heated to $50^{\circ} \mathrm{C}$ for breaking droplets. The broken droplets were then dried using vacuum box, followed by adding DMSO to dissolve CPT. Fluorescent intensities were detected using plate reader system, then the CPT concentration was calculated according to the standard linear calibration curve. Drug loading efficiency (LE) and drug encapsulation efficiency (EE) were calculated by formula below:

$$
\begin{gathered}
\mathrm{LE} \%=(\text { Weight of drug in droplets / Weight of dried } \\
\text { droplets }) \times 100 \%
\end{gathered}
$$

$\mathrm{EE} \%=($ Weight of drug in droplets / Weight of feeding drugs) $x 100 \%$

\section{Particle stability of RBCMDs}

To compare the particle stability of RBCMD with that of the conventional acoustic PEG-droplets prepared by synthetic lipids (polyethylene glycol (PEG)-modified 1,2-distearoyl-OT-glycero-3-phosphocholine (DSPC)) and RBCMDs were adjusted to the same concentration and incubated with $100 \%$ serum at $37^{\circ} \mathrm{C}$. After incubation for the designated time lengths $(0,0.5,1,2,4,12 \& 24$ hour), the droplets were resuspended and examined for the concentration and size by using a Multisizer. Relative droplet concentration $(\%)$ was calculated from dividing the measured droplet concentration by the original droplet concentration.

\section{Macrophage uptake}

Primary mouse peritoneal macrophages were isolated from male C57BL/6 mice (6-8 weeks) following previous published protocols. $1 \times 10^{6}$ of primary mouse peritoneal macrophages per well were seeded in 6-well plates and incubated overnight. $5 \mathrm{x}$ $10^{6}$ of DiO-labeled droplets were added into culture medium and incubated for $10 \mathrm{~min}$. Cocultured macrophages were washed with PBS for 3 times to remove the free droplets. Droplet-uptake macrophages were then trypsinized and examined the fluorescent intensity using flow cytometry.

\section{In vitro B-mode ultrasound imaging}

B-mode ultrasound imaging was performed to investigate the ultrasound contrast enhancing ability of RBCMDs before and after ADV following the previously published experimental setups [29]. A clinical ultrasound imaging system equipped with a 128-element linear array probe (model t 3000 , Terason, MA, USA) was used to detect the echogenicity at an acoustic frequency and mechanical index of 7.5 and 0.7 respectively. Diluted droplets were poured into a cylindrical chamber in an acoustically transparent phantom constructed by $2 \mathrm{wt} \%$ agarose gel (Invitrogen Co.,CA,USA). The beam axis of the imaging probe was aligned perpendicular to the HIFU transducer (SU-107, Sonic Concepts, WA, USA) with the focus positioned above the HIFU sonication site to avoid the interference. The same HIFU pulses with an acoustic frequency of $3.5 \mathrm{MHz}$ and a pulse duration of 3 cycles were used to continuously induce ADV at a pulse repetition frequency of 10 pulses/s. The ultrasound contrast enhancing ability was evaluated in terms of signal to noise ratio (SNR) calculated from decomposed ultrasound data using MATLAB® software (MathWorks, MA, USA).

\section{Anti-cancer efficacy by the combined treat- ment of ultrasound/CPT-RBCMDs}

HeLa cells were seeded in a 96-well plate $\left(5 \times 10^{3}\right.$ per well) overnight. $1 \times 10^{6}$ of droplets were added into each well, filled up with serum-free medium to avoid bubbles between HIFU trigger probe and well-sealed with plastic wrap. To activate the anti-cancer efficacy of CPT-RBCMDs, half of each well area was scanned by HIFU with $8 \mathrm{MPa}$ (peak rarefactional pressure) and 3-cycle pulses to trigger ADV. After the ultrasound treatment, cells were incubated for another 1 hour. Afterwards, cells were washed with PBS twice and replaced with fresh medium. Cell viability was analyzed after 24 hour of $37^{\circ} \mathrm{C} / 5 \% \mathrm{CO}_{2}$ incubation using MTT assay.

\section{Physical cell damage by ADV of RBCMD}

To evaluate the damage caused by physical force through ADV, $3 \times 10^{5}$ of BJAB cells in $300 \mu \mathrm{L}$ culture medium were mixed with RBCMDs $\left(1 \times 10^{9}\right.$ droplets $/ \mathrm{mL}$ ) followed by $3 \mathrm{~min}$ insonation $(3.5 \mathrm{MHz}, 8$ $\mathrm{MPa}$ and 200 pulse/s). After HIFU treatment, cells were collected and re-suspended with fresh medium, followed by MTT assay for 4 hours to estimate cell viability. Optical studies of cell damage through ADV were observed with an integrated acousto-optical system with high speed camera (FASTCAM SA4, Photron, Tokyo, Japan) [29]. BJAB cells with RBCMDs were injected into 200-mm-diameter vessel-mimicking cellulose tube (Spectrum Labs, CA, USA). To observe the images of cell damage through $\mathrm{ADV}$, only one $\mathrm{BJAB}$ cell was insonated by a single 3.5-MHz and 3-cycle HIFU pulse at $8 \mathrm{MPa}$ (peak rarefactional pressure) within the optical field of view.

\section{ADV-triggered drug release and its chemical toxicity}

To measure the active ultrasound triggered drug 
release, CPT-loaded RBCMDs were insonated with 3.5-MHz, 3-cycle, and 8-MPa HIFU at 200 pulses/s for 3 minutes in a tube with an ultrasound-permeable window (Figure 7A). Insonated samples were centrifuged at $2000 \times \mathrm{g}$ for $30 \mathrm{sec}$ immediately to sediment the intact droplets, then the supernatant with released CPT was collected and determined the CPT concentration. For evaluating the anti-cancer efficacy of released CPT, $5 \times 10^{4}$ Hela cells were treated by released CPT solution (1:20 dilution in culture medium). Treated cells were incubated in $37^{\circ} \mathrm{C} \mathrm{CO}_{2}$ incubator for 24 hours, followed by MTT assay for 4 hours to estimate cell viability.

\section{In vivo acoustic vaporization and B-mode im- aging}

The animals were handled in accordance to the animal use protocol of the National Tsing Hua University, Hsinchu, Taiwan. $2.5 \times 10^{8}$ RBCMDs in $50 \mu$ L PBS were retro-orbitally injected into male C57BL mice. The retro-orbital venous sinus is described as a confluence of several vessels. After mice anesthesia, we carefully inserted the needle into mouse medial canthus at an angle of approximately $30^{\circ}$, and slowly push the solution into retro-orbital venous sinus; the detail procedure of retro-orbital injection is shown in [31]. Comparison with the common-used lateral tail vein injection, retro-orbital injection provides higher rate of successful injection that can decrease the stress of mice and there is no difference of pharmacokinetics via both two intravenous injections [32, 33]. After waiting for $10 \mathrm{~min}$ for thorough distribution of RBCMDs in the blood circulation, the right leg of the mice was insonated with $3.5-\mathrm{MHz}$ and 3-cycle HIFU (SU-107, Sonic Concepts, WA, USA) at an acoustic pressure of $8 \mathrm{MPa}$ (peak rarefactional pressure) at 10 pulses/s for $2 \mathrm{~min}$. The ultrasound B-mode images of the leg muscle before and after ADV were collected using a high-end medical ultrasound imaging system (Aplio 500, Toshiba, Ottawa, Japan) with an $18-\mathrm{MHz}$ probe. The images were compared to calculate ultrasound contrast enhancement resulting from ADV bubbles. The left leg of the same mice was also considered as control group without HIFU insonation.

\section{Identification of CD47 on RBCMDs}

For CD47 identification, the resulting gel from SDS-PAGE was transferred to a nitrocellulose membrane. The membrane was then stained with a primary rat anti-mouse CD47 antibody (BD Biosciences) and a secondary goat anti-rat IgG HRP conjugate (GeneTex). Then the membrane was subject to Plus-ECL western blotting substrate (PerkinElmer) and developed with G:Box ChemiXT 16 system (Syngene).

\section{Statistics}

Results of this study are presented as the mean and standard deviation of at least three independent measurements. All statistical evaluations were carried out with unpaired two-tailed Student's t-test. $p$-value of less than 0.05 was considered significant $(p<0.05$, * $\left.; p<0.01,{ }^{* *} ; p<0.001,{ }^{* * *}\right)$.

\section{Supplementary Material}

Additional File 1:

Figure S1-S2 representing the results obtained from CD47 identification on RBCMDs using Western Blot techniques and the leakage test and HIFU-triggered drug release of CPT-loaded RBCMDs respectively. http://www.thno.org/v05p1264s1.pdf

Additional File 2:

Video S1 representing cell integrity damages caused by RBCMD acoustic vaporization.

http://www.thno.org/v05p1264s2.mp4

\section{Acknowledgement}

This work was supported by grants from National Health Research Institutes (NHRI) of Taiwan (NHRI-EX103-10221EC) and Ministry of Science and Technology of Taiwan (NSC102-2113-M-007-006-MY2) and National Tsing Hua University (104N2046E1/104N2732E1).

\section{Competing Interests}

The authors have declared that no competing interest exists.

\section{References}

1. Furman NET, Lupu-Haber Y, Bronshtein T, Kaneti L, Letko N, Weinstein E, et al. Reconstructed stem cell nanoghosts: a natural tumor targeting platform. Nano Lett. 2013; 13: 3248-55.

2. Parodi A, Quattrocchi N, van de Ven AL, Chiappini C, Evangelopoulos M, Martinez JO, et al. Synthetic nanoparticles functionalized with biomimetic leukocyte membranes possess cell-like functions. Nat Nanotechnol. 2013; 8: 61-68.

3. Hu CMJ, Fang RH, Copp J, Luk BT, Zhang LF. A biomimetic nanosponge that absorbs pore-forming toxins. Nat Nanotechnol. 2013; 8: 336-40.

4. Hu CMJ, Zhang L, Aryal S, Cheung C, Fang RH, Zhang LF. Erythrocyte membrane-camouflaged polymeric nanoparticles as a biomimetic delivery platform. P Natl Acad Sci USA. 2011; 108: 10980-85.

5. Hu CMJ, Fang RH, Zhang LF. Erythrocyte-inspired delivery systems. Adv Healthc Mater. 2012; 1: 537-47.

6. Godfrin Y, Horand F, Franco R, Dufour E, Kosenko E, Bax BE, et al. International seminar on the red blood cells as vehicles for drugs. Expert Opin Biol Th. 2012; 12: 127-33.

7. Magnani M. Erythrocytes as carriers for drugs: the transition from the laboratory to the clinic is approaching. Expert Opin Biol Th. 2012; 12: 137-38.

8. Kushner AM, Guan ZB. Modular design in natural and biomimetic soft materials. Angew Chem Int Edit. 2011; 50: 9026-57.

9. Aizenberg J, Fratzl P. Biological and biomimetic materials. Adv Mater. 2009; 21: $387-8$.

10. Lejeune A, Moorjani M, Gicquaud C, Lacroix J, Poyet P, Gaudreault RC. Nanoerythrosome, a new derivative of erythrocyte ghost - preparation and antineoplastic potential as drug carrier for daunorubicin. Anticancer Res. 1994; 14: 915-19.

11. Gao WW, Hu CMJ, Fang RH, Luk BT, Su J, Zhang LF, Surface functionalization of gold nanoparticles with red blood cell membranes. Adv Mater. 2013; 25: 3549-53.

12. Aryal $\mathrm{S}, \mathrm{Hu} \mathrm{CM}$, Fang $\mathrm{RH}$, Dehaini $\mathrm{D}$, Carpenter $\mathrm{C}$, Zhang $\mathrm{DE}$, et al. Erythrocyte membrane-cloaked polymeric nanoparticles for controlled drug loading and release. Nanomedicine. 2013; 8: 1271-80. 
13. Kim H, Lee D, Kim J, Kim TI, Kim WJ. Photothermally triggered cytosolic drug delivery via endosome disruption using a functionalized reduced graphene oxide. ACS Nano. 2013; 7: 6735-46.

14. Luo YL, Shiao YS, Huang YF. Release of photoactivatable drugs from plasmonic nanoparticles for targeted cancer therapy. ACS nano. 2011; 5: 7796-804.

15. Florinas S, Nam HY, Kim SW. Enhanced siRNA delivery using a combination of an arginine-grafted bioreducible polymer, ultrasound, and microbubbles in cancer cells. Mol Pharmaceut. 2013; 10: 2021-30

16. Chiang $\mathrm{WH}, \mathrm{Ho}$ VT, Chen $\mathrm{HH}$, Huang WC, Huang YF, Lin SC, et al Superparamagnetic hollow hybrid nanogels as a potential guidable vehicle system of stimuli-mediated MR imaging and multiple cancer therapeutics. Langmuir. 2013; 29: 6434-43.

17. Kawabata K, Sugita N, Yoshikawa H, Azuma T, Umemura S. Nanoparticles with multiple perfluorocarbons for controllable ultrasonically induced phase shifting. Jpn J Appl Phys 1. 2005; 44: 4548-52.

18. Kripfgans OD, Fowlkes JB, Miller DL, Eldevik OP, Carson PL. Acoustic droplet vaporization for therapeutic and diagnostic applications. Ultrasound Med Biol. 2000; 26: 1177-89.

19. Wang $\mathrm{CH}$, Kang ST, Lee $\mathrm{YH}$, Luo YL, Huang YF, Yeh CK. Aptamer-conjugated and drug-loaded acoustic droplets for ultrasound theranosis. Biomaterials. 2012; 33: 1939-47.

20. Kagan D, Benchimol MJ, Claussen JC, Chuluun-Erdene E, Esener S, Wang J. Acoustic droplet vaporization and propulsion of perfluorocarbon-loaded microbullets for targeted tissue penetration and deformation. Angew Chem Int Ed Engl. 2012; 51: 7519-22.

21. Rapoport NY, Kennedy AM, Shea JE, Scaife CL, Nam KH. Controlled and targeted tumor chemotherapy by ultrasound-activated nanoemulsions/microbubbles. J Control Release. 2009; 138: 268-76.

22. Javadi M, Pitt WG, Tracy CM, Barrow JR, Willardson BM, Hartley JM, et al. Ultrasonic gene and drug delivery using eLiposomes. J Control Release. 2013; 167: 92-100.

23. Zhu ML, Jiang LX, Fabiilli ML, Zhang AL, Fowlkes JB, Xu LX. Treatment of murine tumors using acoustic droplet vaporization-enhanced high intensity focused ultrasound. Phys Med Biol. 2013; 58: 6179-91.

24. Oldenborg PA, Zheleznyak A, Fang YF, Lagenaur CF, Gresham HD, Lindberg FP. Role of CD47 as a marker of self on red blood cells. Science. 2000; 288: 2051-2054

25. Giovanella BC, Hinz HR, Kozielski AJ, Stehlin JS, Silber R, Potmesil M. Complete growth-inhibition of human cancer xenografts in nude-mice by treatment with 20-(S)-camptothecin. Cancer Res. 1991; 51: 3052-55.

26. Fan $\mathrm{CH}$, Ting $\mathrm{CY}$, Liu HL, Huang $\mathrm{CY}$, Hsieh $\mathrm{HY}$, Yen $\mathrm{TC}$, et al. Antiangiogenic-targeting drug-loaded microbubbles combined with focused ultrasound for glioma treatment. Biomaterials. 2013; 34: 2142-55.

27. Wong ZZ, Kripfgans OD, Qamar A, Fowlkes JB, Bull JL. Bubble evolution in acoustic droplet vaporization at physiological temperature via ultra-high speed imaging. Soft Matter. 2011; 7: 4009-16

28. Sheeran PS, Wong VP, Luois S, McFarland RJ, Ross WD, Feingold S, et al. Decafluorobutane as a phase-change contrast agent for low-energy extravascular ultrasonic imaging. Ultrasound in Medicine and Biology. 2011; 37: $1518-30$

29. Kang ST, Yeh CK. Intracellular acoustic droplet vaporization in a single peritoneal macrophage for drug delivery applications. Langmuir. 2011; 27: 13183-88.

30. Feshitan JA, Chen CC, Kwan JJ, Borden MA. Microbubble size isolation by differential centrifugation. J Colloid Interf Sci. 2009; 329: 316-24.

31. Yardeni T, Eckhaus $M$, Morris HD, Huizing $M$, Hoogstraten-Miller S. Retro-orbital injections in mice. Lab Anim. 2011; 40: 155-60.

32. Steel CD, Stephens AL, Hahto SM, Singletary SI, Ciavarra RP. Comparison of the lateral tail vein and the retro-orbital venous sinus as routes of intravenous drug delivery in a transgenic mouse model. Lab Anim. 2008; 37: 26-32.

33. Schoch A, Thorey IS, Engert J, Winter G, Emrich T. Comparison of the lateral tail vein and the retro-orbital venous sinus routes of antibody administration in pharmacokinetic studies. Lab Anim. 2014; 43: 95-99. 